\title{
Interface conditions for degenerate two-phase flow equations in one space dimension
}

\author{
F. Buzzi, M. Lenzinger and B. Schweizer
}

Preprint 2009-02

Januar 2009

Fakultät für Mathematik

Technische Universität Dortmund

Vogelpothsweg 87

44227 Dortmund 



\title{
Interface conditions for degenerate two-phase flow equations in one space dimension
}

\author{
Fulvia Buzzi, Michael Lenzinger and Ben Schweizer
}

January 19, 2009

\begin{abstract}
Summary: We study the two-phase flow equations describing, e.g., the motion of oil and water in a porous material, and are concerned with interior interfaces where two different porous media are in contact. At such an interface, the entry pressure relation together with the degeneracy of the system leads to an interesting effect known as oil-trapping. Restricting to the one-dimensional case we show an existence result with the help of appropriate regularizations and a time discretization. The crucial tool is a compactness lemma: The control of the time derivative in a space of measures is used to conclude the strong convergence of a sequence.
\end{abstract}

\section{Introduction}

We consider the motion of two immiscible fluids in a porous medium. Denoting the fluids with indices $j=1,2$, it is standard to use the variables of pressure and saturation, $p_{j}$ and $u_{j}$. Interpreting the saturations as volume fractions in the pore space, we have $u:=u_{1}=1-u_{2}$. Conservation of mass for each phase together with Darcy's law yield the system of two-phase flow equations

$$
\begin{aligned}
\partial_{t} u & =\nabla \cdot\left(k(x) k_{r, 1}(u) \nabla p_{1}\right), \\
-\partial_{t} u & =\nabla \cdot\left(k(x) k_{r, 2}(u) \nabla p_{2}\right),
\end{aligned}
$$

where the various coefficients $k$ stand for the absolute and relative permeabilities. The equations are completed by the capillary pressure relation $p_{c}(u)=p_{1}-p_{2}$. Adding the equations yields

$$
\nabla \cdot\left(K(x, u) \nabla p_{1}-k(x) k_{r, 2}(u) \nabla\left[p_{c}(u)\right]\right)=0
$$

where $K(x, u):=k(x)\left(k_{r, 1}(u)+k_{r, 2}(u)\right)$.

We restrict to the one-dimensional case $x \in(-L, L)$. In this special situation the elliptic equation (1.3) implies that the total flux is constant in space,

$$
K(x, u) \partial_{x} p_{1}-k(x) k_{r, 2}(u) \partial_{x}\left[p_{c}(x, u)\right]=-q_{0} .
$$

AMS 2000 subject classification: 35K65, 76T99

Key words: two-phase flow, porous media, degenerate diffusion, transmission condition 
We assume for simplicity that the flux of oil and water at the left boundary are prescribed. In this case, also the value of the total flux $q_{0}$ is determined by the boundary conditions. Setting $k_{i}(x, u):=k(x) k_{r, i}(u)$ we can solve (1.4) for $\partial_{x} p_{1}$,

$$
\partial_{x} p_{1}=-q_{0} \frac{1}{K(x, u)}+\frac{k_{2}(x, u)}{K(x, u)} \partial_{x}\left[p_{c}(x, u)\right]
$$

insert into (1.1) and obtain

$$
\begin{aligned}
\partial_{t} u+\partial_{x} F & =0 \\
F & =f(u)-k(x) \lambda_{r}(u) \partial_{x}\left[p_{c}(x, u)\right],
\end{aligned}
$$

with

$$
f(u):=q_{0} \frac{k_{r, 1}(u)}{k_{r, 1}(u)+k_{r, 2}(u)}, \quad \lambda_{r}(u):=\frac{k_{r, 1}(u) k_{r, 2}(u)}{k_{r, 1}(u)+k_{r, 2}(u)} .
$$

Equation (1.5) is the standard approach for the modelling of two-phase flow in soil or rock and hence of utmost importance in applications. At the same time, the equations are mathematically challenging due to a double degeneracy. One regards the vanishing permeability $k_{r, 1}(s) \rightarrow 0$ for a vanishing saturation $s$, leading to

$$
f(s) \rightarrow 0 \text { and } \lambda_{r}(s) \rightarrow 0 \text { for } s \rightarrow 0 .
$$

Small diffusion can create free boundaries between wet and dry regions, an effect that appears also in the standardized porous media equation $\partial_{t} u=\Delta\left(u^{2}\right)$ or in thin film flow, see $[4,8]$ and the references therein. Technically, a vanishing permeability inhibits the derivation of uniform estimates for the gradients of the pressure.

A second degeneracy regards the capillary pressure $p_{c}$, which can have infinite slope and should be regarded as a multi-valued function. Formally, this degeneracy can be regarded as an infinite diffusion and leads to elliptic-parabolic equations, see [2, 14]. For us, this fact implies that already the choice of the primary variable is a problem. Above, we formulated relations for the saturation $u$. This has the advantage that, physically, a saturation is defined everywhere, while a pressure can be defined only where fluid is present, i.e. for $u>0$. The mathematical counterpart of this observation is that $p_{c}$ is multi-valued at $u=0$. When we write the above system in the pressure variable $v$ with $u=b(v):=p_{c}^{-1}(v)$, then the degeneracy $b^{\prime}(v)=0$ (for some $v$ ) leads, at least formally, to $\partial_{t} u=0$ and thus to an elliptic system. The physical initial and boundary conditions typically involve both quantities, the initial condition prescribes a saturation and the boundary condition prescribes a pressure. We refer to [13] and the references therein for the modelling of boundary conditions.

Despite the double degeneracy of the equations, existence results are available. For one-phase flow equations (Richards equation) we refer to [2, 3, 15], for the two-phase flow equations to $[1,6,7,11,12]$. Most of these contributions use a formulation in pressure variables. The contributions of the authors regard $x$-dependent coefficients or outflow boundary conditions that require to use the saturation variable in a substantial way. 

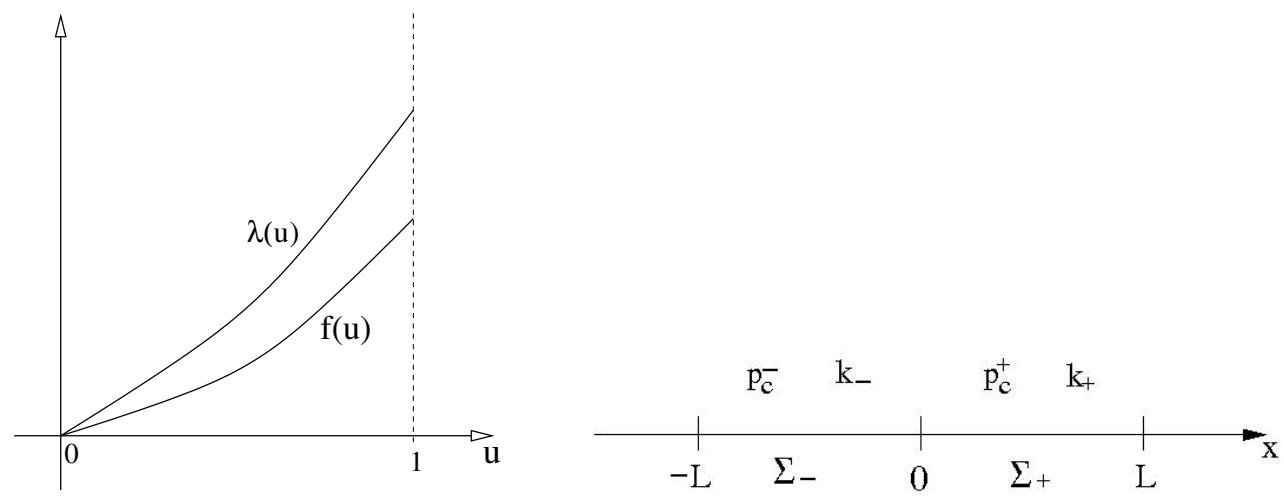

Figure 1: Left: Qualitative behavior of the coefficient functions $f(x, u)$ and $\lambda(x, u)$ which are degenerate at $u=0$. Right: The flow domain $\Omega=(-L, L)$. The left part $\Sigma_{-}$contains another material than the right part $\Sigma_{+}$(e.g. a fine and a coarse medium).

\section{Interior interfaces}

The equations become even more interesting when interior interfaces are involved. To analyze a single interface we set $\Sigma_{-}:=(-L, 0)$ for the fine material and $\Sigma_{+}:=(0, L)$ for the coarse material. We assume that the material parameters are constant in each medium, but different in the two materials. We write $k^{ \pm}$and $p_{c}^{ \pm}$for the permeability and capillary pressure on $\Sigma_{ \pm}$. We set $\Sigma:=\Sigma_{-} \cup \Sigma_{+}$and $\Sigma_{T}:=\Sigma \times(0, T)$, see Figure 1 .

We assume

$$
k(x):=\left\{\begin{array}{ll}
k^{+} & \text {for } \quad x \in \Sigma_{+} \\
k^{-} & \text {for } \quad x \in \Sigma_{-}
\end{array} \quad p_{c}(x, u):=\left\{\begin{array}{lll}
p_{c}^{+}(u) & \text { for } & x \in \Sigma_{+} \\
p_{c}^{-}(u) & \text { for } & x \in \Sigma_{-} .
\end{array}\right.\right.
$$

and write, accordingly, $\lambda(x, u):=k(x) \lambda_{r}(u)$ and $f(x, u)=f^{ \pm}(u)$.

We will often work with a pressure variable $v$ such that $u=\left(p_{c}\right)^{-1}(v)$. For calculations in the two variables we set $\tilde{\lambda}:=\lambda \circ p_{c}^{-1}$ and denote by $\tilde{\Lambda}(x, v)$ a primitive of $\tilde{\lambda}(x, v)$, $\tilde{\Lambda}^{\prime}(x, v):=\partial_{v} \tilde{\Lambda}(x, v)=\tilde{\lambda}(x, v)$, normalized by $\tilde{\Lambda}(x, 0)=0$ for all $x \in \Sigma$. We further set $\Lambda:=\tilde{\Lambda} \circ p_{c}$ on $(0,1)$, extended continuously to $[0,1]$. We emphasize that $\Lambda^{\prime} \neq \lambda$. With these definitions, at least formally, the diffusive part of the flux $F$ can be written in various forms,

$$
\lambda(u) \partial_{x}\left[p_{c}(u)\right]=\tilde{\lambda}(v) \partial_{x} v=\tilde{\Lambda}^{\prime}(v) \partial_{x} v=\partial_{x}[\tilde{\Lambda}(v)]=\partial_{x}[\Lambda(u)] .
$$

in $\Sigma \times(0, T)$. We include the warning that the function $\Lambda(x, s)$ will, in general, have a jump in $x=0$. This implies that the above relations do not hold in the whole domain $\Omega_{T}$, i.e. across $x=0$.

Transmission conditions. At the interface $x=0$ the physical interpretation suggests to use as the two transmission conditions the continuity of the flux and the continuity of the capillary pressure. We use the notation $h\left(0^{ \pm}\right)=\lim _{\delta \backslash 0} h(0 \pm \delta)$.

Continuity of the flux. The conservation of mass requires that the fluxes on the lefthand side and the right-hand side with respect to $x=0$ are balanced. Therefore, the first 

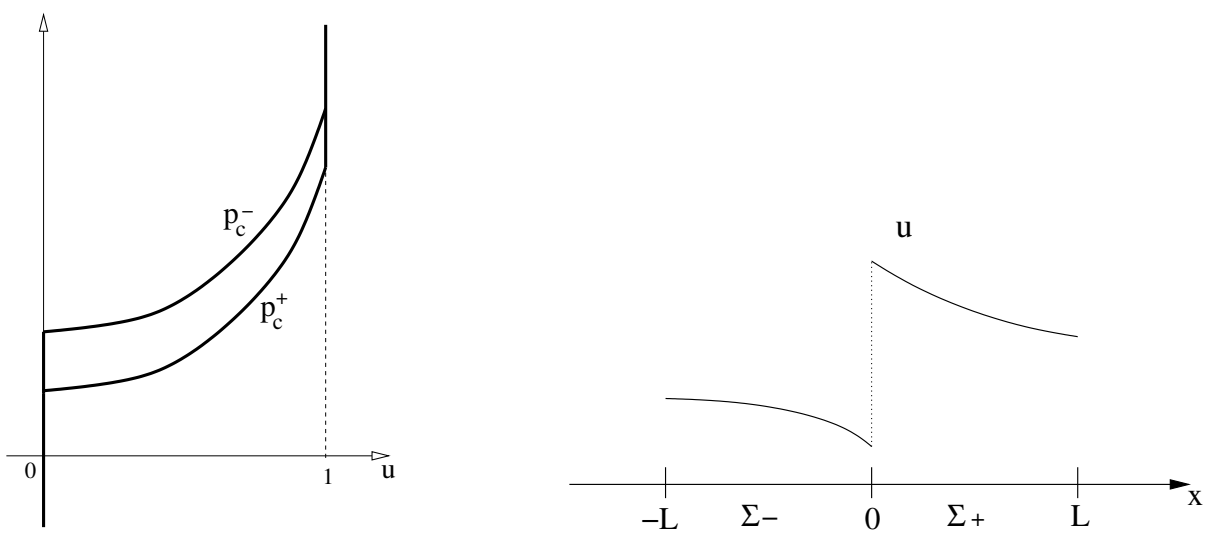

Figure 2: Left: The set-valued functions $p_{c}^{+}$and $p_{c}^{-}$. Right: A possible saturation in $\Sigma$, saturations $u\left(0^{+}\right)$and $u\left(0^{-}\right)$as in case (i). The saturation jumps in $x=0$ as a consequence of the continuity of flux and capillary pressure.

condition reads

$$
F\left(0^{-}, u\left(0^{-}\right)\right)=F\left(0^{+}, u\left(0^{+}\right)\right) .
$$

Continuity of the capillary pressure. One may think of the pressures $p_{1}$ and $p_{2}$ as continuous functions across $x=0$. We will therefore demand the continuity of the capillary pressure $p_{c}(x, u(x, t))=p_{1}-p_{2}$ at $x=0$. This implies that the saturation is discontiuous, see Figure 2.

We define the minimal pressures in $\Sigma_{-}$and $\Sigma_{+}$in the limit of a vanishing saturation as

$$
p_{\min }^{ \pm}:=\lim _{s \searrow 0} p_{c}^{ \pm}(s), \quad p_{\max }^{ \pm}:=\lim _{s \nearrow 1} p_{c}^{ \pm}(s) .
$$

The capillary pressures $p_{c}^{ \pm}$should be regarded as set-valued functions defined as the maximal monotone graphs given by

$$
\begin{aligned}
& p_{c}^{ \pm}(u)=\left\{\tilde{p}_{c}^{ \pm}(u)\right\} \text { for } u \in(0,1), \\
& p_{c}^{ \pm}(0)=\left(-\infty, p_{\min }^{ \pm}\right] \text {and } p_{c}^{ \pm}(1)=\left[p_{\max }^{ \pm}, \infty\right)
\end{aligned}
$$

where $\tilde{p}_{c}^{ \pm}$are monotonically increasing real-valued functions satisfying (1.7). The continuity condition for the capillary pressure then reads

$$
p_{c}^{-}\left(u\left(0^{-}\right)\right) \cap p_{c}^{+}\left(u\left(0^{+}\right)\right) \neq \emptyset .
$$

We concentrate from now on on the vanishing saturation $u=0$.

Let us illustrate condition (1.8) with an example. Assuming $p_{\min }^{+} \leq p_{\min }^{-}$, the condition demands

$$
\begin{array}{cl}
p_{c}^{-}\left(u\left(0^{-}\right)\right)=p_{c}^{+}\left(u\left(0^{+}\right)\right) & \text {if } u\left(0^{+}\right)>0, \\
p_{c}^{-}\left(u\left(0^{-}\right)\right) \leq p_{\min }^{-} & \text {if } u\left(0^{+}\right)=0
\end{array}
$$

Regarding the effect of oil-trapping, we can additionally define a residual oil saturation $u^{*}$ by $p_{c}^{+}\left(u^{*}\right)=p_{\min }^{-}$and abbreviate $u^{+}:=u\left(0^{+}\right)$and $u^{-}:=u\left(0^{-}\right)$. The capillary pressure 
$p_{c}$ is continuous in the sense of (1.8) if one of the following possibilities occurs.

(i) $\quad u^{+}>u^{*}$ and $u^{-}$satisfies $p_{c}^{-}\left(u^{-}\right)=p_{c}^{+}\left(u^{+}\right)$

(ii) $u^{+} \leq u^{*}$ and $u^{-}=0$.

Here, we identified $p_{c}^{ \pm}$with the real-valued functions $\tilde{p}_{c}^{ \pm}$wherever the first is singlevalued. The effect of oil trapping appears when no oil is present on the left hand side, $u^{-}=0$. Then the degeneracy of $k^{-}$inhibits the transport of oil. On the right hand side, an oil saturation $u^{*}$ is trapped. This phenomenon is studied e.g. in $[9,10,16]$. Regarding the interface conditions we refer to $[5,13]$.

\section{Main result}

We study the following equations for the saturation $u$ and the flux $F$.

$$
\begin{aligned}
& \partial_{t} u+\partial_{x} F=0 \quad \text { in } \Sigma_{T}, \\
& F(x, u)=f(x, u)-\lambda(x, u) \partial_{x}\left[p_{c}(u)\right] \text { in } \Sigma_{T}, \\
& F \text { and } p_{c}(x, u) \text { are continuous in } x=0,
\end{aligned}
$$

the continuity of $p_{c}$ in the sense of (1.8). For system (1.10) we impose, for given measurable initial saturation $u_{0}:(-L, L) \rightarrow[0,1]$ and pressure boundary values $p_{0} \in W^{1,1}((0, T), \mathbb{R})$ with $p_{\min }^{+}<p_{0}(t)<p_{\max }^{+}$for all $t$, the following initial and boundary conditions

$$
\begin{aligned}
\left.u\right|_{t=0} & =u_{0} \text { on }(-L, L) \\
F(-L, t) & =0 \text { and } p_{c}(u(L, t))=p_{0}(t) \text { for all } t \in(0, T) .
\end{aligned}
$$

We emphasize that our methods are not restricted to this choice of boundary conditions.

We assume that the coefficient functions satisfy the following regularity and monotonicity properties.

$$
\begin{aligned}
& f^{ \pm} \in C^{0}([0,1], \mathbb{R}), \lambda^{ \pm} \in C^{0,1}([0,1], \mathbb{R}), p_{c}^{ \pm} \in C^{1}([0,1], \mathbb{R}) \\
& f^{ \pm}(0)=\lambda^{ \pm}(0)=0, \quad f^{ \pm}, \lambda^{ \pm}>0 \text { on }(0,1], \\
& f^{ \pm} \leq c_{1} \lambda^{ \pm} \text {and }\left(p_{c}^{ \pm}\right)^{\prime} \geq c_{2} \text { on }[0,1] \text { for constants } c_{1}, c_{2}>0 .
\end{aligned}
$$

The following definition makes our notion of solutions precise.

Definition 1.1. A function $u \in L^{\infty}\left(\Omega_{T},[0,1]\right)$ is called $a$ weak solution of system (1.10) in $\Omega_{T}=(-L, L) \times(0, T)$ iffor a flux function $F \in L^{2}\left(\Omega_{T}\right)$

$$
\begin{array}{rlrl}
\partial_{t} u+\partial_{x} F & =0 & & \text { in } \mathcal{D}^{\prime}\left(\Omega_{T}\right), \\
F(u) & =f(u)-\partial_{x}[\Lambda(u)] & \text { in } \mathcal{D}^{\prime}\left(\Sigma_{T}\right), \\
p_{c}^{-}\left(u\left(0^{-}\right)\right) & \cap p_{c}^{+}\left(u\left(0^{+}\right)\right) \neq \emptyset & \text { for a.e. } t .
\end{array}
$$

For the last relation we note that $\Lambda(u)$ has a trace by (1.14) which can be used to evaluate $u$ in boundary points. We say that $u$ satisfies the initial and boundary conditions (1.11) if

$$
\int_{\Omega_{T}} u \partial_{t} \varphi+\int_{\Omega_{T}} F \partial_{x} \varphi=-\int_{\Omega} u_{0} \varphi(0)
$$


for all $\varphi \in C_{0}^{\infty}([-L, L) \times[0, T))$. The Dirichlet boundary condition is demanded in the sense of traces,

$$
p_{c}(u(L, t))=p_{0}(t)
$$

using again the existence of traces for $u$.

In order to evaluate point-values of $u$ we use the extended inverse of $\Lambda$, the function $\Lambda^{-1}: \mathbb{R} \rightarrow[0,1]$ which is continuous and constant (equal to 0 or 1 ) beyond its natural domain of definition $\left[\tilde{\Lambda}\left(p_{\min }\right), \tilde{\Lambda}\left(p_{\max }\right)\right]$.

We note that the continuity of the flux $F$ is included through the equation which contains $\partial_{x} F$. Our main result is the following existence statement.

Theorem 1.2. Let assumptions (1.12) hold. Then there exists a weak solution u of system (1.10) with boundary conditions (1.11).

We will show the existence with the help of a regularized system. The degenerate coefficient $\lambda$ prevents us from showing an $L^{2} H^{1}$-estimate for the pressure. Our main tool will be a powerful compactness lemma.

There are two possible regularization approaches. One, performed here, replaces the degenerate coefficients by regularized coefficients that allow a priori estimates. At the same time, the jump discontinuity at the interface is kept. A second approach would be to regularize additionally the jump discontinuity, i.e. to replace the coefficients by smooth functions in $x$. This second approach was carried out in [5] and can be regarded also as a justification of the transmission conditions.

The proof of our main result proceeds in three stages. In Section 2 we define the regularization of the coefficients with a parameter $\eta>0$ and show the existence of a solution for the regularized problem with a time discretization. In Section 3 we derive the necessary a priori estimates to perform the limit $\eta \rightarrow 0$ and to find limit functions $u$ and $F$ satisfying the equation and the interface condition. The compactness lemma 1.3 is crucial in this step. The lemma is shown in Appendix A.

Comparison to the literature. We note that the very general results of [2] can not be applied directly to our problem, since the function $b(x, \cdot)=p_{c}(x, \cdot)^{-1}$ has an $x$-dependence, which is even non-smooth. The same comment concerns the results of [6] (compare $\gamma_{1} \in L^{\infty} L^{2}$ in assumption (A5), where $\gamma_{1}$ contains $\partial_{x} p_{c}$ ). We emphasize that the two quoted articles concern the higher dimensional case.

An existence result for the above equations in the one-dimensional case appeared in [16], but in that work a monotonicity property of solutions was exploited, i.e. the special structure of initial and boundary conditions was used. Another existence result was given by the interesting contribution of Bertsch, Passo and Duijn [5], where the flux is chosen as an independent variable. This choice has the advantage that, like the pressure, the flux has no jumps. Similar to hyperbolic estimates, the authors derive $L^{\infty} B V$ estimates for the flux, which implies a gain of regularity (at the cost of assuming initial values with $\left(u_{0}^{2}\right)^{\prime} \in B V(\mathbb{R} \backslash\{0\})$, see assumption (H) of [5]). The flux estimates exploit that the coefficient functions $f$ and $\lambda_{r}$ are $x$-independent and linearly affine in both media (see the relation before (1.6) in [5]). Our approach is more direct and yields a more general existence statement. Nevertheless, our results do not cover $x$-regularized coefficients. 
Compactness Lemma. The following lemma provides a compactness result in $L^{2}\left(\Omega_{T}\right)$ for a bounded family of functions $u_{k} \in L^{2}\left(0, T ; H^{1}(\Omega)\right)$, which additionally has a uniform bound for the time derivatives $\partial_{t} u_{k}$ in $\left(C^{0}\left(0, T ; H_{0}^{1}(\Omega)\right)^{\prime}\right.$. We believe that this compactness statement is no surprise to experts, but we are not aware of a formulation in the literature.

Lemma 1.3 (Compactness). Let $\Omega \subset \mathbb{R}^{N}$ be bounded and $T>0$. Let $\left(u_{k}\right)_{k \in \mathbb{N}}$ be a bounded sequence in $L^{2}\left(0, T ; H^{1}(\Omega)\right)$ such that

$$
\partial_{t} u_{k} \in\left(C^{0}\left(0, T ; H_{0}^{1}(\Omega)\right)^{\prime}\right.
$$

is bounded independent of $k$. Then there exists a subsequence $u_{k}$ and a limit $u$ such that

$$
u_{k} \rightarrow u \text { in } L^{2}\left(0, T ; L^{2}(\Omega)\right) \text { for } k \rightarrow \infty \text {. }
$$

We will apply the lemma in Section 3 to the solutions $u_{\eta}$ of a regularized problem. The compactness is the essential tool to derive the solution property for weak limits. Due to the degeneracy of the equation we can not derive the typical $L^{2}\left(0, T ; H^{1}(\Omega)\right) \cap$ $H^{1}\left(0, T ; H^{-1}(\Omega)\right)$ estimates which provides the compactness with the lemma of LionsAubin.

Regarding notation we agree that $C$ denotes real constants in estimates; their value may change from one inequality to the next. We use squared brackets such as $\partial_{x}[f(u)]$ to denote the $x$ derivative of the function $f(x, u(x))$. To indicate norms we sometimes abbreviate function spaces with time variable and write, e.g., $L^{2} H^{1}$ instead of $L^{2}\left(0, T ; H^{1}(\Omega)\right)$.

\section{Regularized equations}

In this section we define a regularized system of non-degenerate equations and show the existence of solutions to this regularized problem.

Definition 2.1. For a sequence $0<\eta \rightarrow 0$ we define the family of regularized coefficient functions $f_{\eta}^{ \pm}, \lambda_{\eta}^{ \pm}, p_{c, \eta}^{ \pm}$as follows.

$$
f_{\eta}^{ \pm}, \lambda_{\eta}^{ \pm} \in C^{1}([0,1],[0, \infty)), \quad p_{c, \eta}^{ \pm} \in C^{2}((0,1), \mathbb{R})
$$

with $p_{c, \eta}^{ \pm}(s) \rightarrow-\infty$ for $s \rightarrow 0$ and $p_{c, \eta}^{ \pm}(s)=+\infty$ for $s \rightarrow 1$. The regularized coefficients $f_{\eta}^{ \pm}, \lambda_{\eta}^{ \pm}, p_{c, \eta}^{ \pm}$are chosen such that $\lambda_{\eta}^{ \pm} \geq \eta>0$ with $f_{\eta}^{ \pm} \leq c_{1} \lambda_{\eta}^{ \pm}$and $\left(p_{c, \eta}^{ \pm}\right)^{\prime} \geq c_{2}$. Concerning the critical saturations we demand that $\lambda_{\eta}^{ \pm} \equiv \lambda^{ \pm}(1)$ on $[1-\eta, 1]$, and that $p_{c, \eta}^{ \pm}(\eta) \geq p_{\min }^{0}:=\min \left\{p_{\min }^{+}, p_{\min }^{-}\right\}$and $p_{c, \eta}^{ \pm}(1-\eta)=p_{\max }^{ \pm}$. We demand the convergence

$$
\begin{aligned}
& \lambda_{\eta}^{ \pm} \rightarrow \lambda^{ \pm}, f_{\eta}^{ \pm} \rightarrow f^{ \pm} \quad \text { uniformly in }[0,1], \\
& p_{c, \eta}^{ \pm} \rightarrow p_{c}^{ \pm} \quad \text { uniformly on compact subsets of }(0,1) .
\end{aligned}
$$

Finally, the initial saturation is regularized to a smooth function $u_{0, \eta}:[-L, L] \rightarrow[\eta, 1-$ $\eta]$ such that $u_{0, \eta} \rightarrow u_{0}$ in $L^{1}((-L, L))$. 


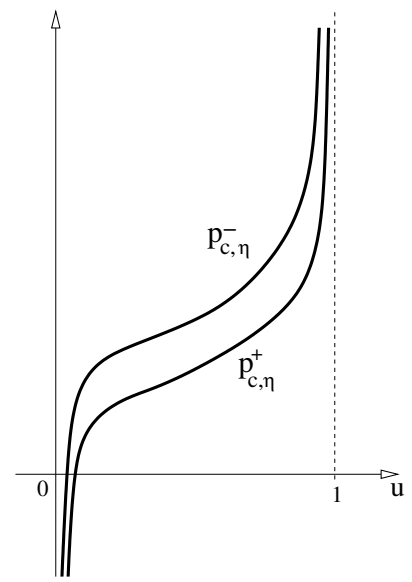

Figure 3: Regularized capillary pressures $p_{c, \eta}^{ \pm}$for $\eta>0$.

In the sequel we will additionally use $\tilde{f}_{\eta}=f_{\eta} \circ p_{c, \eta}^{-1}, \tilde{\lambda}_{\eta}=\lambda_{\eta} \circ p_{c, \eta}^{-1}$ and $\tilde{\Lambda}_{\eta}$ as the primitive of $\tilde{\lambda}_{\eta}$ with $\tilde{\Lambda}_{\eta}^{ \pm}(0)=0,\left(\tilde{\Lambda}_{\eta}^{ \pm}\right)^{\prime}=\tilde{\lambda}_{\eta}^{ \pm}$. Furthermore, we define $\Lambda_{\eta}=\tilde{\Lambda}_{\eta} \circ p_{c, \eta}$ on $(0,1)$.

We emphasize that for any $\eta>0$ we demand the special $x$-dependence $f_{\eta}(x, u)=$ $f_{\eta}^{ \pm}(u)$ for $x \in \Sigma_{ \pm}$, and the same for $\lambda_{\eta}, \Lambda_{\eta}$ and $p_{c, \eta}$. The functions $\tilde{\Lambda}_{\eta}$ and $\Lambda$ are strictly monotonically increasing which allows to construct the inverse functions. We have the uniform convergence $\left(p_{c, \eta}\right)^{-1} \rightarrow\left(p_{c}\right)^{-1}$ on $\mathbb{R}$ and $\tilde{\Lambda}_{\eta} \rightarrow \tilde{\Lambda}$ uniformly on compact subsets of $\mathbb{R}$.

In order to treat the boundary conditions of Neumann type on the left and of Dirichlet type on the right, we set

$$
H_{*}^{1}(\Omega):=\left\{w \in H^{1}(\Omega): w(L)=0\right\} \text { and } H_{*}^{-1}(\Omega):=\left(H_{*}^{1}(\Omega)\right)^{\prime} .
$$

Lemma 2.2 (Existence for the regularized problem). For regularized coefficients as in Definition 2.1, there exists a weak solution $u_{\eta} \in H^{1}\left(0, T ; H_{*}^{-1}(\Omega)\right) \cap L^{\infty}\left(\Omega_{T}\right)$ of $(1.10)$ with initial and boundary data $u_{0, \eta}$ and $p_{0}$. We can give a quite strong formulation of the equation: with $v_{\eta}:=p_{c, \eta}\left(u_{\eta}\right) \in L^{2}\left(0, T ; H^{1}(\Omega)\right)$ and $F_{\eta}:=f_{\eta}\left(u_{\eta}\right)-\lambda_{\eta}\left(u_{\eta}\right) \partial_{x} v_{\eta}$ holds

$$
\int_{\Omega_{T}} \partial_{t} u_{\eta} \varphi-\int_{\Omega_{T}} F_{\eta} \partial_{x} \varphi=0
$$

for all $\varphi \in L^{2}\left((0, T) ; H_{*}^{1}(\Omega)\right)$, and the boundary conditions $v_{\eta}(L, t)=p_{0}(t)$ and $u_{\eta}(0)=$ $u_{0, \eta}$ hold in the sense of traces.

The transmission condition for the pressure is contained with the strong statement $v_{\eta} \in L^{2}\left(0, T ; H^{1}(\Omega)\right)$.

Proof. Step 1. Preliminaries. We use $b:=p_{c, \eta}^{-1}:(-\infty, \infty) \rightarrow[0,1]$ and suppress the subscript $\eta$ in the following. We note that $\tilde{f}(v)=f_{\eta}(b(v)) \leq c_{1} \lambda_{\eta}(b(v))=c_{1} \tilde{\lambda}(v)$ by our assumptions. Our aim is to construct a solution $v \in L^{2}\left(0, T ; H^{1}(\Omega)\right)$ of

$$
\partial_{t}[b(v)]+\partial_{x}\left[\tilde{f}(v)-\tilde{\lambda}(v) \partial_{x} v\right]=0 \quad \text { in } \Omega_{T}
$$


with $v(., 0)=v_{0}:=p_{c, \eta}\left(u_{0, \eta}\right)$ and $v(L, t)=p_{0}(t)$. By a density argument, in order to verify (2.2), it is sufficient to solve (2.3) in the sense of distributions for test-functions $\varphi \in C_{0}^{\infty}\left((0, T) ; C_{0}^{\infty}([-L, L))\right.$. Our choice of the regularization asserts for the initial condition the uniform lower bound $v_{0}=p_{c, \eta}\left(u_{0, \eta}\right) \geq p_{c, \eta}(\eta) \geq p_{\min }^{0}$.

Step 2. Time discretization. We use a small parameter $\delta>0$ and construct approximate solutions with a time discretization. We discretize (2.3) in time, setting $\delta:=\Delta t$, $t_{k}:=k \delta$ and $K \delta=T, k=0,1, \ldots, K$. In each time step $k \leq K-1$ we solve

$$
\frac{b\left(v_{k+1}\right)-b\left(v_{k}\right)}{\delta}+\partial_{x}\left[\tilde{f}\left(v_{k+1}\right)-\tilde{\lambda}\left(v_{k+1}\right) \partial_{x} v_{k+1}\right]=0
$$

with initial condition $v_{0}=p_{c, \eta}\left(u_{0}\right)$ and boundary conditions $v_{k+1}(L)=p_{0}\left(t_{k+1}\right)$ and $\left[\tilde{f}\left(v_{k+1}\right)-\tilde{\lambda}\left(v_{k+1}\right) \partial_{x} v_{k+1}\right](-L)=0$. The existence of a solution $v_{k+1} \in H^{1}(\Omega)$ of the discrete problem follows e.g. with variational methods by the monotonicity of $b$ and with an iteration. An application of a maximum principle with stationary comparison solutions implies a uniform bound $\left\|v_{k}\right\|_{L^{\infty}} \leq \rho_{0}\left(p_{0}, p_{\min }^{0}, p_{\max }^{0}, c_{1}\right)$ for all $k$, independent of $\eta$ and $\delta$. For this step, it is important to note that a stationary solution $V$ with $\partial_{x}(\tilde{f}(V)-$ $\left.\left.\partial_{x} \tilde{\Lambda}(V)\right]\right)=0$ satisfies, for our boundary condition, $\partial_{x} V=\tilde{f}(V) / \tilde{\lambda}(V) \leq c_{1}$, and is therefore bounded. Regarding to problems in the transmission point we refer to a similar maximum principle in [16].

Step 3. Energy estimates. Our intention is to multiply equation (2.4) with $v_{k+1}-p_{0}$. We define $\Phi$ as the primitive of $p_{c, \eta}=b^{-1}$ satisfying $\Phi(b(0))=0$ such that $\Phi(x, z)=$ $\Phi^{ \pm}(z)$ in $\Sigma^{ \pm}$. Let $s_{k}(x):=b\left(v_{k}(x)\right)$ and $\hat{s}$ the corresponding linear interpolation of the functions $s_{k}$. With the notation $\hat{v}:=b^{-1}(\hat{s})$ we obtain, in the interval $\left(t_{k}, t_{k+1}\right)$,

$$
\partial_{t} \Phi(\hat{s})=\Phi^{\prime}(\hat{s}) \frac{s_{k+1}-s_{k}}{\delta}=\hat{v} \partial_{t} \hat{s}
$$

and therefore

$$
\int_{\Omega_{T}} \partial_{t} \hat{s} v_{k+1}=\int_{\Omega_{T}} \partial_{t} \hat{s} \hat{v}+\int_{\Omega_{T}} \partial_{t} \hat{s}\left(v_{k+1}-\hat{v}\right) \geq \int_{\Omega_{T}} \partial_{t} \Phi(\hat{s}),
$$

where the positivity of the third integral is a consequence of the monotonicity of $b$. We introduce, additionally to the interpolation $\hat{v}$, the interpolation $\bar{v}$ with $\bar{v}(t)=v_{k+1}$ for all $t \in\left(t_{k}, t_{k+1}\right)$, and similarly $\bar{p}_{0}$. Multiplication of (2.4) with $v_{k+1}-p_{0}\left(t_{k+1}\right)$ then yields

$$
\int_{\Omega_{T}} \partial_{t} \Phi(\hat{s})+\int_{\Omega_{T}} \tilde{\lambda}(\bar{v})\left|\partial_{x} \bar{v}\right|^{2} \leq \int_{\Omega_{T}} \tilde{f}(\bar{v}) \partial_{x} \bar{v}+\int_{\Omega_{T}} \partial_{t} \hat{s} \bar{p}_{0} .
$$

We write the second integral on the right-hand side of (2.7) as

$$
\int_{\Omega_{T}} \partial_{t} \hat{s} \bar{p}_{0}=-\int_{\Omega_{T}} s_{k} \frac{p_{0}\left(t_{k+1}\right)-p_{0}\left(t_{k}\right)}{\delta}+s_{K} p_{0}\left(t_{K}\right)-u_{0, \eta} p_{0}(0) .
$$

Using the boundedness of $s_{k}$ and $p_{0}\left(t_{k}\right)$ and the inequality $\sum_{k}\left|p_{0}\left(t_{k+1}\right)-p_{0}\left(t_{k}\right)\right| \leq$ $\left\|\partial_{t} p_{0}\right\|_{L^{1}}$, we see that the second integral on the right hand side of inequality (2.7) is bounded. The other integral can be absorbed into the left hand side and we obtain, by $\tilde{\lambda} \geq \eta$, a uniform bound for $\|\hat{v}\|_{L^{2} H^{1}} \leq C$, with $C$ depending on $\eta$, but independent of $\delta$. 
Step 4. Compactness and limit equation. The evolution equation (2.4) yields the bound $\left\|\partial_{t} \hat{s}\right\|_{L^{2} H_{*}^{-1}} \leq C$ with $C$ depending on $\eta$, but independent of $\delta$. The spatial regularity on each subdomain $\Sigma_{ \pm}$can be checked directly with $\hat{s}=b(\hat{v})$,

$$
\int_{0}^{T} \int_{\Sigma_{+}}\left|\partial_{x} \hat{s}\right|^{2}=\int_{0}^{T} \int_{\Sigma_{+}}\left|b^{\prime}(\hat{v})\right|^{2}\left|\partial_{x} \hat{v}\right|^{2} \leq C
$$

with $C$ independent of $\delta$. The lemma of Lions-Aubin states that the embedding $L^{2} H^{1} \cap$ $H^{1} H^{-1} \subset L^{2} L^{2}$ is compact. We can therefore extract a strongly convergent subsequence $\hat{s}=\hat{s}_{\delta} \rightarrow s$ strongly in $L^{2} L^{2}$ for $\delta \rightarrow 0$. The maximum principle for $v_{k}$ implies bounds $\hat{s} \in[\varepsilon, 1-\varepsilon]$, with $\varepsilon>0$ depending on $\eta$ (due to the infinite slope of $p_{c, \eta}$ ), but independent of $\delta$. Since $p_{c, \eta}$ is continuous on $[\varepsilon, 1-\varepsilon]$, we conclude additionally the strong convergence of the pressure, $\hat{v}=\hat{v}_{\delta}=p_{c, \eta}\left(\hat{s}_{\delta}\right) \rightarrow v$ strongly in $L^{2}\left(0, T ; L^{2}(\Omega)\right)$ for $\delta \rightarrow 0$.

The strong convergences permit to evaluate limits of the nonlinear coefficient functions. To compare the piecewise constant interpolation $\bar{v}$ with the piecewise linear interpolation $\hat{v}$ we use a general comparison result for interpolations (see [12], Lemma 3.2): The strong convergence of $\hat{s}_{\delta}$ implies the strong convergence $\bar{s}_{\delta} \rightarrow s$ in $L^{2}\left(0, T ; L^{2}(\Omega)\right)$ for $\delta \rightarrow 0$. This carries over to the convergence $\bar{v}_{\delta} \rightarrow \bar{v}$. After choosing a further subsequence, the convergences $\tilde{f}\left(\bar{v}_{\delta}\right) \rightarrow \tilde{f}(v)$ and $\tilde{\lambda}\left(\bar{v}_{\delta}\right) \rightarrow \tilde{\lambda}(v)$ in $L^{2}\left(0, T ; L^{2}(\Omega)\right)$ hold true. This allows to pass to the limit in the time discrete equation (2.4) and to obtain equation (2.3) for $v$.

\section{The degenerate equations}

The aim of this section is to perform the limit $\eta \rightarrow 0$. The difficulty is that the $L^{2} H^{1}$ estimate for $v$ was dependent on $\eta$. Furthermore, in the compactness argument of the last section, we used a priori bounds for the saturation away from the critical points 0 and 1. These bounds were also $\eta$-dependent and therefore the compactness of the sequence $u_{\eta}$ in $L^{2} L^{2}$ will require additional arguments. We will use the compactness result of Lemma 1.3 to take the limit $u_{\eta} \rightarrow u$ and conclude that $u$ satisfies the original degenerate equations and the transmission condition for multi-valued functions $p_{c}^{ \pm}$. With this section we conclude the existence result of Theorem 1.2.

A priori estimates. We use the same energy estimates as in the last proof, i.e. estimate (2.7) for the limit $v_{\eta}$. For convenience we derive the estimate again from the solution property. Using $\varphi=v_{\eta}-p_{0}=p_{c, \eta}\left(u_{\eta}\right)-p_{0}$ in (2.2) we obtain

$$
\int_{\Omega_{T}} p_{c, \eta}\left(u_{\eta}\right) \partial_{t} u_{\eta}+\int_{\Omega_{T}} \tilde{\lambda}\left(v_{\eta}\right)\left|\partial_{x} v_{\eta}\right|^{2} \leq \int_{\Omega_{T}} \tilde{f}\left(v_{\eta}\right) \partial_{x} v_{\eta}+\int_{\Omega_{T}} \partial_{t} u_{\eta} p_{0} .
$$

Using again the primitive $\Phi_{\eta}$ of $p_{c, \eta}$ with $\Phi_{\eta}(b(0))=0$ we write the first integral as a total time derivative, $\partial_{t} \Phi_{\eta}\left(u_{\eta}\right)=p_{c, \eta}\left(u_{\eta}\right) \partial_{t} u_{\eta}$. With the $\eta$-independent bound for $p_{0} \in$ $W^{1,1}((0, T))$ and $u_{\eta} \in L^{\infty}$ we treat the last integral. The uniform estimate $\tilde{f}^{ \pm} \leq c_{1} \tilde{\lambda}^{ \pm}$ allows to absorb the first integral. Since $\Phi_{\eta}\left(u_{0, \eta}\right)$ is bounded and $\Phi_{\eta}\left(u_{\eta}\right)$ is positive, we conclude

$$
\int_{\Sigma_{T}} \lambda_{\eta}\left(u_{\eta}\right)\left(p_{c, \eta}^{\prime}\left(u_{\eta}\right)\right)^{2}\left|\partial_{x} u_{\eta}\right|^{2} \leq C
$$


with $C$ independent of $\eta$. We have only a $\Sigma$-integral, since the chain rule evaluation of $\partial_{x} v_{\eta}$ is valid only away from $x=0$. We can express this estimate, with arbitrary $\delta>0$, with cut-off functions. Using that $p_{c, \eta}^{\prime} \geq c_{2}$ is bounded from below and $\lambda_{\eta}^{ \pm}(s) \geq c_{\delta}$ for all $s \in[\delta, 1]$ for some $c_{\delta}>0$, we conclude

$$
\int_{\Omega_{T}}\left|\partial_{x} u_{\eta}\right|^{2} \mathbf{1}_{\left\{u_{\eta} \geq \delta\right\}} \mathbf{1}_{\Sigma} \leq C_{\delta}
$$

where $C_{\delta}$ depends on $\delta$ but is independent of $\eta ; \mathbf{1}_{A}$ denotes the characteristic function of the set $A$. From the estimate (3.2) and the boundedness of $f_{\eta}$ and $\lambda_{\eta}$ we additionally conclude that the family $F_{\eta}=\left[f_{\eta}\left(u_{\eta}\right)-\lambda_{\eta}\left(u_{\eta}\right) p_{c, \eta}^{\prime}\left(u_{\eta}\right) \partial_{x} u_{\eta}\right] \mathbf{1}_{\Sigma}$ is uniformly bounded in $L^{2}\left(\Omega_{T}\right)$, i.e.

$$
\left\|F_{\eta}\right\|_{L^{2}\left(\Omega_{T}\right)} \leq C
$$

with $C$ independent of $\eta$.

Compactness. Since $\left(u_{\eta}\right)_{\eta>0}$ is uniformly bounded in $L^{2}\left(\Omega_{T}\right)$ there exists a weakly convergent subsequence $u_{\eta} \rightarrow u$ in $L^{2}\left(\Omega_{T}\right)$ for $\eta \rightarrow 0$. Our aim is to show strong convergence.

Claim 1: Strong convergence.

$$
u_{\eta} \rightarrow u \text { strongly in } L^{2}\left(\Omega_{T}\right) \text { for } \eta \rightarrow 0 .
$$

Our aim is to apply Lemma 1.3 to a modified $u_{\eta}$. We fix an arbitrary $\delta>0$ and define

$$
\phi_{\delta}(\xi):= \begin{cases}0 & \text { for } \xi<\delta \\ \tilde{\phi}_{\delta}(\xi) & \text { for } \delta \leq \xi<3 \delta \\ \xi-2 \delta & \text { for } \xi \geq 3 \delta\end{cases}
$$

where $\tilde{\phi}_{\delta}:[\delta, 3 \delta] \rightarrow \mathbb{R}$ is chosen such that $\phi_{\delta}$ is smooth with $0 \leq \tilde{\phi}_{\delta}^{\prime} \leq 1$. Since $\phi_{\delta}^{\prime}\left(u_{\eta}\right)=0$ for $u_{\eta}<\delta$ and $\phi_{\delta}^{\prime}\left(u_{\eta}\right) \leq 1$ for all $u_{\eta}$, using estimate (3.3) we obtain that $\partial_{x}\left[\phi_{\delta}\left(u_{\eta}\right)\right]=\phi_{\delta}^{\prime}\left(u_{\eta}\right) \partial_{x} u_{\eta}$ is uniformly bounded in $L^{2}\left(\Sigma_{T}\right)$. Hence, $\phi_{\delta}\left(u_{\eta}\right)$ is uniformly bounded in $L^{2}\left(0, T ; H^{1}(\Sigma)\right)$. We note that the derivatives $\phi_{\delta}^{\prime}\left(u_{\eta}\right)$ and $\phi_{\delta}^{\prime \prime}\left(u_{\eta}\right)$ are bounded, in particular $\phi_{\delta}^{\prime \prime}\left(u_{\eta}\right)=0$ for $u_{\eta}<\delta$.

With the help of (3.3) and (3.4) we are now able to show the uniform boundedness of $\partial_{t}\left[\phi_{\delta}\left(u_{\eta}\right)\right]$ in $\left(C^{0}\left(0, T ; H_{0}^{1}(\Omega)\right)^{\prime}\right.$. For any $\varphi \in C^{0}\left(0, T ; H_{0}^{1}(\Omega)\right)$ we calculate

$$
\begin{aligned}
\int_{\Sigma_{T}} \partial_{t}\left[\phi_{\delta}\left(u_{\eta}\right)\right] \varphi & =\int_{\Sigma_{T}} \phi_{\delta}^{\prime}\left(u_{\eta}\right) \partial_{t} u_{\eta} \varphi=\int_{\Sigma_{T}}-\partial_{x} F_{\eta} \phi_{\delta}^{\prime}\left(u_{\eta}\right) \varphi=\int_{\Sigma_{T}} F_{\eta} \partial_{x}\left[\phi_{\delta}^{\prime}\left(u_{\eta}\right) \varphi\right] \\
& =\int_{\Sigma_{T}} F_{\eta} \phi_{\delta}^{\prime \prime}\left(u_{\eta}\right) \partial_{x} u_{\eta} \varphi+\int_{\Sigma_{T}} F_{\eta} \phi_{\delta}^{\prime}\left(u_{\eta}\right) \partial_{x} \varphi \\
& \leq\|\varphi\|_{C^{0} H^{1}}\left\|F_{\eta}\right\|_{L^{2} L^{2}}\left\|\phi_{\delta}^{\prime \prime}\left(u_{\eta}\right) \partial_{x} u_{\eta}\right\|_{L^{2} L^{2}}+\left\|\partial_{x} \varphi\right\|_{C^{0} L^{2}}\left\|F_{\eta}\right\|_{L^{2} L^{2}} \\
& \leq C_{\delta}\|\varphi\|_{C^{0} H^{1}} \quad \text { for all } \eta .
\end{aligned}
$$

Lemma 1.3 implies the strong convergence of a subsequence of $w_{\eta, \delta}:=\phi_{\delta}\left(u_{\eta}\right)$ in $L^{2}\left(\Omega_{T}\right)$, i.e. $w_{\eta, \delta} \rightarrow w_{\delta}$ for $\eta \rightarrow 0$ and for all $\delta>0$. 
We define $U_{\eta, \delta}:=u_{\eta}-w_{\eta, \delta}$ and consider $u_{\eta}=U_{\eta, \delta}+w_{\eta, \delta}$. Since $w_{\eta, \delta}$ is a Cauchy sequence in $L^{2}\left(\Omega_{T}\right)$ and $\left|U_{\eta, \delta}\right| \leq 2 \delta$ for all $\eta$ we deduce that also $u_{\eta}$ is a Cauchy sequence in $L^{2}\left(\Omega_{T}\right)$. Therefore, we finally obtain

$$
u_{\eta} \rightarrow u \quad \text { in } \quad L^{2}\left(\Omega_{T}\right) .
$$

Furthermore, exploiting the maximum principle, we have the convergence $v_{\eta} \stackrel{*}{\rightarrow} v$ in $L^{\infty}\left(\Omega_{T}\right)$.

Claim 2: Identification of limits. We claim that, along a subsequence,

$$
\begin{aligned}
& b_{\eta}\left(v_{\eta}\right) \rightarrow u=b(v) \text { strongly in } L^{2}\left(\Omega_{T}\right), \\
& \tilde{\Lambda}_{\eta}\left(v_{\eta}\right) \rightarrow \tilde{\Lambda}(v) \text { weakly in } L^{2}\left(0, T ; H^{1}(\Sigma)\right) .
\end{aligned}
$$

In the proof of the claim we do not emphasize $x$-dependence of the coefficients and make separate calculation on $\Sigma_{ \pm}$, suppressing the superscripts \pm . The convergence $u_{\eta}=$ $b_{\eta}\left(v_{\eta}\right) \rightarrow u$ was already shown. To identify the limit, we use $\delta>0$ and a continuous function $\psi_{\delta}:[0,1] \rightarrow[0,1]$ with $\psi_{\delta}(s)=0$ for $s$ in a neighborhood of $\{0,1\}$ and $\psi_{\delta}(s)=1$ for $s \in[\delta, 1-\delta]$. We have

$$
\psi_{\delta}\left(u_{\eta}\right) v_{\eta}=\psi_{\delta}\left(u_{\eta}\right) p_{c, \eta}\left(u_{\eta}\right) \rightarrow \psi_{\delta}(u) p_{c}(u),
$$

since, on compact subsets of $(0,1)$ the convergence $p_{c, \eta} \rightarrow p_{c}$ is uniform. The left hand side converges weakly to $\psi_{\delta}(u) v$. Since $\delta>0$ was arbitrary, we conclude $v=p_{c}(u)$ almost everywhere on $\{u(.) \in(0,1)\}$. Concerning the set of points with $u=1$ we calculate, with $\psi_{\delta}(s)=0$ for $s<1-\delta$ and $\psi_{\delta}(1)=1$,

$$
\psi_{\delta}\left(u_{\eta}\right)\left(v_{\eta}-p_{\max }\right)=\psi_{\delta}\left(u_{\eta}\right)\left(p_{c, \eta}\left(u_{\eta}\right)-p_{\max }\right) \geq O(\delta),
$$

hence $v \geq p_{\max }$ on $\{u()=1$.$\} . Similarly, we find v \leq p_{\min }$ on $\{u()=0$.$\} and hence$ $v \in p_{c}(u)$ almost everywhere. This shows (3.6).

The calculation for $\tilde{\Lambda}$ is slightly different since $\tilde{\Lambda}$ is not constant above $p_{\max }$. Nevertheless, the maximum principle for $v_{\eta}$ implies boundedness of $\tilde{\Lambda}_{\eta}\left(v_{\eta}\right)$ in $L^{\infty}$ and in $L^{2} H^{1}$, and we may assume $\tilde{\Lambda}_{\eta}\left(v_{\eta}\right) \rightarrow U$ weakly in $L^{2} H^{1}$. We now exploit the linear behavior $\tilde{\Lambda}_{\eta}(t)=\alpha_{\eta}+\tilde{\lambda}(1)\left(t-p_{\max }\right)$ for $t \geq p_{\max }$, which holds for some sequence $\alpha_{\eta} \rightarrow \alpha \in \mathbb{R}$. We use a cut-off function $\tilde{\psi}_{\delta}: \mathbb{R} \rightarrow[0,1]$ satisfying $\tilde{\psi}_{\delta}(t)=0$ for $t \leq p_{\max }$ and $\tilde{\psi}_{\delta}(t)=1$ for $t>p_{\max }+\delta$. As for the nonlinear function $b$, we find $\tilde{\psi}_{\delta}\left(v_{\eta}\right) \rightarrow \tilde{\psi}_{\delta}(v)$ strongly in $L^{2}$ and therefore

$$
\tilde{\psi}_{\delta}(v) U \leftarrow \tilde{\psi}_{\delta}\left(v_{\eta}\right) \tilde{\Lambda}_{\eta}\left(v_{\eta}\right) \rightarrow \tilde{\psi}_{\delta}(v) \tilde{\Lambda}(v) \text { weakly in } L^{2}
$$

the second convergence holds since $\tilde{\Lambda}$ is a linear function on the relevant arguments. We find the characterization $U=\tilde{\Lambda}(v)$ almost everywhere on $\{u=1\}$. The identification on the other parts is done as before, exploiting the uniform bounds for the pressure and the uniform convergence $\tilde{\Lambda}_{\eta} \rightarrow \tilde{\Lambda}$ uniformly on compact subsets of $\mathbb{R}$. Relation (3.7) and Claim 2 follow. 
Limit equations. We fix a subsequence with $F_{\eta} \rightarrow F$ in $L^{2}\left(\Omega_{T}\right)$ and $\tilde{\Lambda}_{\eta}\left(v_{\eta}\right) \rightarrow \tilde{\Lambda}(v)=$ $\Lambda(u)$ in $L^{2}\left(\Omega_{T}\right)$. The limit functions $u$ and $F$ satisfy the equation $\partial_{t} u+\partial_{x} F=0$ in $\mathcal{D}^{\prime}\left(\Omega_{T}\right)$. Furthermore, we obtain for all $\varphi \in C_{0}^{\infty}\left(\Sigma_{T}\right)$

$$
\int_{\Sigma_{T}} F \varphi \leftarrow \int_{\Sigma_{T}} F_{\eta} \varphi=\int_{\Sigma_{T}} f_{\eta}\left(u_{\eta}\right) \varphi+\tilde{\Lambda}_{\eta}\left(v_{\eta}\right) \partial_{x} \varphi \rightarrow \int_{\Sigma_{T}} f(u) \varphi+\Lambda(u) \partial_{x} \varphi .
$$

This implies equation (1.14) for the flux function $F$.

Concerning the initial condition and the flux condition at $x=-L$ we obtain, using the convergences of $u_{\eta}$ and $F_{\eta}$ in $L^{2}\left(\Omega_{T}\right)$,

$$
\begin{aligned}
-\int_{\Sigma} u_{\eta}(0) \varphi(0) & =\int_{0}^{T} \int_{\Sigma} \partial_{t} u_{\eta} \varphi+\int_{0}^{T} \int_{\Sigma} u_{\eta} \partial_{t} \varphi \\
& =\int_{0}^{T} \int_{\Sigma} F_{\eta} \partial_{x} \varphi+\int_{0}^{T} \int_{\Sigma} u_{\eta} \partial_{t} \varphi \rightarrow \int_{\Omega_{T}} F \partial_{x} \varphi+\int_{\Omega_{T}} u \partial_{t} \varphi,
\end{aligned}
$$

for all $\varphi \in C_{0}^{\infty}([-L, L) \times[0, T))$. The regularized solutions satisfy the initial condition $u_{\eta}(0)=u_{0, \eta} \rightarrow u_{0}$ in $L^{1}\left(\Omega_{T}\right)$ which implies (1.16).

Concerning the Dirichlet boundary condition (1.17) we recall that $\tilde{\Lambda}_{\eta}^{ \pm} \rightarrow \tilde{\Lambda}^{ \pm}$uniformly and that $\tilde{\Lambda}^{ \pm}$is invertible on the interval $\left[\min _{t} p_{0}(t), \max _{t} p_{0}(t)\right] \subset\left(p_{\min }^{+}, p_{\max }^{+}\right)$. The convergence $\tilde{\Lambda}_{\eta}\left(v_{\eta}\right) \rightarrow \tilde{\Lambda}(v)$ in $L^{2}\left(0, T, H^{1}\left(\Sigma_{+}\right)\right)$implies for the traces

$$
\tilde{\Lambda}^{+}\left(p_{0}(t)\right) \leftarrow \tilde{\Lambda}_{\eta}^{+}\left(p_{0}(t)\right)=\tilde{\Lambda}_{\eta}^{+}\left(v_{\eta}(L, t)\right) \rightarrow \tilde{\Lambda}^{+}(v)(L, t)
$$

in $L^{2}(0, T)$ for $\eta \rightarrow 0$. Therefore we have $v(L, t)=p_{0}(t)$ a.e. in $(0, T)$.

Transmission condition at the interface. In order to define the traces $u\left(0^{ \pm}\right)$at both sides of the interface we abbreviate $\xi:=\Lambda^{ \pm}(u) \in L^{2}\left((0, T) ; H^{1}\left(\Sigma_{ \pm}\right)\right)$and set $\xi^{ \pm}:=\xi\left(0^{ \pm}\right) \in$ $L^{2}((0, T))$. According to our agreement in Definition 1.1 we set $u\left(0^{ \pm}\right):=\left(\Lambda^{ \pm}\right)^{-1}\left(\xi^{ \pm}\right)$ and define $\mu_{ \pm}:=v\left(0^{ \pm}\right):=\left(\tilde{\Lambda}^{ \pm}\right)^{-1}\left(\xi^{ \pm}\right)$in $L^{2}((0, T))$. Since $\Lambda^{-1}=p_{c}^{-1} \circ \tilde{\Lambda}^{-1}$ we also have $u\left(0^{ \pm}\right)=p_{c}^{-1}\left(\mu_{ \pm}\right)$for a.e. $t$.

In the following calculations we assume $p_{\min }^{+} \leq p_{\min }^{-}$as in Figure 2, in the opposite case the result follows with the analogous calculation. We introduce the piecewise linear cut-off function $G_{\delta}: \mathbb{R} \rightarrow[0, \infty)$,

$$
G_{\delta}(v):= \begin{cases}0 & \text { for } v \leq p_{\min }^{-}+\delta, \\ v-\left(p_{\min }^{-}+\delta\right) & \text { for } v>p_{\min }^{-}+\delta .\end{cases}
$$

Due to estimate (3.2) we have $G_{\delta}\left(v_{\eta}\right) \in L^{2}\left((0, T), H^{1}(\Omega)\right)$ uniformly in $\eta$. Therefore, for some function $g \in L^{2} H^{1}$ we have $G_{\delta}\left(v_{\eta}\right) \rightarrow g$ in $L^{2} H^{1}$ as $\eta \rightarrow 0$ and we can identify the limit $g=G_{\delta}(v)$ by the same arguments as in Claim 2 above.

We claim that the weak transmission condition

$$
G_{\delta}\left(\mu_{+}(t)\right)=G_{\delta}\left(\mu_{-}(t)\right) \text { for all } \delta>0 \text { and a.e. } t \in(0, T)
$$

holds. Since $G_{\delta}(v) \in L^{2}\left(0, T ; H^{1}(\Omega)\right)$ we have the equality $G_{\delta}(v)\left(0^{-}\right)=G_{\delta}(v)\left(0^{+}\right)$of the traces of $G_{\delta}(v)$ on both sides of the interface. It remains to verify $G_{\delta}\left(v\left(0^{ \pm}\right)\right)=$ 
$G_{\delta}(v)\left(0^{ \pm}\right)$to conclude (3.9). Using the Lipschitz continuous functions $\Psi^{ \pm}=G_{\delta} \circ$ $\left(\tilde{\Lambda}^{ \pm}\right)^{-1}$, we calculate

$$
G_{\delta}\left(v\left(0^{ \pm}\right)\right)=\Psi^{ \pm}\left(\xi\left(0^{ \pm}\right)\right)=\left(\Psi^{ \pm} \circ \xi\right)\left(0^{ \pm}\right)=\left(G_{\delta} \circ\left(\tilde{\Lambda}^{ \pm}\right)^{-1} \circ \xi\right)\left(0^{ \pm}\right) .
$$

With this, we have verified (3.9).

The original transmission condition (1.15) is now easily derived from (3.9). For fixed $t \in(0, T)$ we set $\mu^{ \pm}=\mu_{ \pm}(t)$. We distinguish two cases, (i) $G_{\delta}\left(\mu^{+}\right)=G_{\delta}\left(\mu^{-}\right)>0$ for some $\delta>0$ and (ii) $G_{\delta}\left(\mu_{+}\right)=G_{\delta}\left(\mu_{-}\right)=0$ for all $\delta>0$. In case (i) we obtain $\mu^{+}=\mu^{-}$, hence $u\left(0^{ \pm}\right)=p_{c}^{-1}\left(\mu_{ \pm}\right)>0$ and $p_{c}^{+}\left(u\left(0^{+}\right)\right)=\mu^{+}=\mu^{-}=p_{c}^{-}\left(u\left(0^{-}\right)\right)$. In case (ii) we have $\mu^{ \pm} \leq p_{\text {min }}^{-}$which implies $u\left(0^{-}\right)=0$ and $p_{c}^{+}\left(u\left(0^{+}\right)\right) \ni \mu^{+} \in\left(-\infty, p_{\text {min }}^{-}\right]=$ $p_{c}^{-}\left(u\left(0^{-}\right)\right)$. In particular, the intersection $p_{c}^{+}\left(u\left(0^{+}\right)\right) \cap p_{c}^{-}\left(u\left(0^{-}\right)\right)$is not empty. In both cases the transmission condition (1.15) follows and Theorem 1.2 is shown.

\section{A Proof of the compactness lemma}

We will show in this appendix the compactness lemma 1.3. It regards a bouned spacetime domain $(0, T) \times \Omega \subset \mathbb{R}^{1+N}$ and a family of functions $u_{k}:(0, T) \times \Omega \rightarrow \mathbb{R}$, with $k \in \mathbb{N}$. We repeat the statement for convenience.

Let $\left(u_{k}\right)_{k \in \mathbb{N}}$ and its distributional time derivative satisfy that

$$
u_{k} \in L^{2}\left(0, T ; H^{1}(\Omega)\right) \text { and } \partial_{t} u_{k} \in\left(C^{0}\left(0, T ; H_{0}^{1}(\Omega)\right)^{\prime}\right.
$$

are bounded independent of $k$. Then there exists a subsequence $u_{k}$ and a limit $u$ such that

$$
u_{k} \rightarrow u \text { in } L^{2}\left(0, T ; L^{2}(\Omega)\right) \text { for } k \rightarrow \infty
$$

Proof. At first, we find a subsequence such that

$$
\begin{gathered}
u_{k} \rightarrow u \text { in } L^{2}\left(0, T ; H^{1}(\Omega)\right), \\
\partial_{t} u_{k} \stackrel{*}{\rightarrow} \partial_{t} u \text { in }\left(C^{0}\left(0, T ; H_{0}^{1}(\Omega)\right)^{\prime} .\right.
\end{gathered}
$$

We may assume $u=0$, passing to the sequence $\tilde{u}_{k}=u_{k}-u$ if necessary. Furthermore, due to the density of smooth functions, we can assume that the functions $u_{k}$ are smooth.

Step 1. We provide an estimate of BV-type for the sequence $u_{k}$. For $(p, q) \subset(0, T)$ we define

$$
\|v\|_{B V\left(p, q ; H^{-1}\right)}:=\sup \sum_{m=1}^{M}\left\|v\left(t_{m}^{+}\right)-v\left(t_{m}^{-}\right)\right\|_{H^{-1}(\Omega)},
$$

where the supremum is taken over all $M \in \mathbb{N}$ and all families $t_{m}^{ \pm} \in(p, q), p<t_{1}^{-}<t_{1}^{+}<$ $t_{2}^{-}<t_{2}^{+}<\ldots<t_{M}^{+}<q$. We claim that for a constant $C>0$ there holds the estimate

$$
\left\|u_{k}\right\|_{B V\left(0, T, H^{-1}\right)} \leq C
$$


Let $M \in \mathbb{N}, t_{m}^{ \pm} \in(0, T), m=1, \ldots, M$ be fixed, and $\left(\varphi_{m}\right)_{m=1, \ldots, M}$ a family of functions in $H_{0}^{1}(\Omega)$ with $\left\|\varphi_{m}\right\|_{H^{1}} \leq 1$ for all $m$. For $\delta>0$ sufficiently small we choose an interpolation $\varphi^{\delta} \in C^{0}\left(0, T ; H_{0}^{1}(\Omega)\right)$ with the following properties:

$$
\begin{aligned}
& \varphi^{\delta} \equiv \varphi_{m} \quad \text { in }\left[t_{m}^{-}, t_{m}^{+}\right] \quad \text { for all } m \leq M \\
& \varphi^{\delta} \equiv 0 \quad \text { in }\left[t_{m}^{+}+\delta, t_{m+1}^{-}-\delta\right] \quad \text { for all } m \leq M-1, \\
& \left\|\varphi^{\delta}\right\|_{L^{\infty} H^{1}} \leq 1
\end{aligned}
$$

Due to the boundedness of $\partial_{t} u_{k}$ in $\left(C^{0} H_{0}^{1}\right)^{\prime}$ we obtain

$$
\begin{aligned}
\sum_{m=1}^{M}\left[u_{k}\left(t_{m}^{+}\right)-u_{k}\left(t_{m}^{-}\right)\right]\left(\varphi_{m}\right) & =\sum_{m=1}^{M} \int_{t_{m}^{-}}^{t_{m}^{+}}\left[\partial_{t} u_{k}\right]\left(\varphi_{m}\right)=\lim _{\delta \rightarrow 0} \int_{0}^{T}\left[\partial_{t} u_{k}\right]\left(\varphi^{\delta}\right) \\
& \leq\left\|\partial_{t} u_{k}\right\|_{\left(C^{0} H_{0}^{1}\right)^{\prime}} \cdot\left\|\varphi^{\delta}\right\|_{C^{0} H^{1}} \leq C .
\end{aligned}
$$

Thus (1.1) is proved.

Step 2. For $J \in \mathbb{N}$ large, we divide $(0, T)$ in subintervals $I_{j}, j=1, \ldots, J$ of length $\eta=T / J$. Furthermore, we choose a number $\rho>0$ and define the set of intervals $\mathcal{G}_{k}$ with large values by

$$
j \in \mathcal{G}_{k}: \Longleftrightarrow\left\|u_{k}\right\|_{B V\left(I_{j} ; H^{-1}\right)} \geq \rho .
$$

We can estimate the number of indices $j \in \mathcal{G}_{k}$ as follows,

$$
\left|\mathcal{G}_{k}\right| \cdot \rho \leq \sum_{j \in \mathcal{G}_{k}}\left\|u_{k}\right\|_{B V\left(I_{j} ; H^{-1}\right)} \leq\left\|u_{k}\right\|_{B V\left(0, T ; H^{-1}\right)} \leq C .
$$

Step 3. We estimate variations from mean values over time intervals by the $B V$ seminorm. For intervals $I \subset(0, T), u: I \rightarrow H^{-1}$ and a fixed time $t_{0} \in I$ we calculate the Bochner integrals

$$
\begin{aligned}
\left\|f_{I} u-u\left(t_{0}\right)\right\|_{H^{-1}} & =\left\|\frac{1}{|I|} \int_{I} u(t)-u\left(t_{0}\right) d t\right\|_{H^{-1}} \leq \frac{1}{|I|} \int_{I}\left\|u(t)-u\left(t_{0}\right)\right\|_{H^{-1}} d t \\
& \leq\|u\|_{B V\left(I ; H^{-1}\right)} .
\end{aligned}
$$

Therefore, inserting an arbitrary value $u\left(t_{0}\right)$, we obtain

$$
\begin{aligned}
\int_{I}\left\|u-f_{I} u\right\|_{H^{-1}}^{2} d t & \leq 2 \int_{I}\left\|u-u\left(t_{0}\right)\right\|_{H^{-1}}^{2} d t+2 \int_{I}\left\|u\left(t_{0}\right)-f_{I} u\right\|_{H^{-1}}^{2} d t \\
& \leq 4|I|\|u\|_{B V\left(I ; H^{-1}\right)}^{2} .
\end{aligned}
$$

Step 4. We estimate the $L^{2}\left(\Omega_{T}\right)$-norm of $u_{k}$. Using the interpolation $\|w\|_{L^{2}}^{2} \leq$ $h\|w\|_{H^{1}}^{2}+C_{h}\|w\|_{H^{-1}}^{2}$ for $w \in H^{1}(\Omega)$ we have

$$
\left\|u_{k}\right\|_{L^{2} L^{2}}^{2}=\int_{0}^{T}\left\|u_{k}(t)\right\|_{L^{2}}^{2} d t \leq h \int_{0}^{T}\left\|u_{k}\right\|_{H^{1}}^{2} d t+C_{h} \int_{0}^{T}\left\|u_{k}\right\|_{H^{-1}}^{2} d t .
$$


By insertion of mean values on subintervals we obtain

$$
\begin{gathered}
\left\|u_{k}\right\|_{L^{2} L^{2}}^{2} \leq C h+2 C_{h} \sum_{j \leq J}\left|I_{j}\right|\left\|f_{I_{j}} u_{k}\right\|_{H^{-1}}^{2}+2 C_{h} \sum_{j \in \mathcal{G}_{k}} \int_{I_{j}}\left\|u_{k}-f_{I_{j}} u_{k}\right\|_{H^{-1}}^{2} d t \\
+2 C_{h} \sum_{j \notin \mathcal{G}_{k}} \int_{I_{j}}\left\|u_{k}-f_{I_{j}} u_{k}\right\|_{H^{-1}}^{2} d t .
\end{gathered}
$$

Extracting a subsequence $k \rightarrow \infty$, due to the compactness of $H^{1}(\Omega) \hookrightarrow H^{-1}(\Omega)$, we can assume that

$$
\left\|f_{I_{j}} u_{k}\right\|_{H^{-1}} \rightarrow 0 \text { as } k \rightarrow \infty \text { for all } j=1, \ldots, J .
$$

Therefore, the second term on the right-hand side of (1.2) vanishes for $k \rightarrow \infty$. For the remaining sums we use the $B V$-estimates and obtain for arbitrary $\varepsilon>0$

$$
\left\|u_{k}\right\|_{L^{2} L^{2}}^{2} \leq C h+\frac{\varepsilon}{4}+C_{h} C \frac{\eta}{\rho}+8 C_{h} \rho^{2} .
$$

Given $\varepsilon$, we first choose $h$, then $\rho$ and finally $\eta$ sufficiently small to achieve

$$
\left\|u_{k}\right\|_{L^{2} L^{2}}^{2} \leq \varepsilon
$$

for $k$ sufficiently large. This concludes the proof of the Lemma.

\section{References}

[1] H. W. Alt and E. DiBenedetto. Nonsteady flow of water and oil through inhomogeneous porous media. Ann. Scuola Norm. Sup. Pisa Cl. Sci. (4), 12(3):335-392, 1985.

[2] H.W. Alt and S. Luckhaus. Quasilinear elliptic-parabolic differential equations. Math. Z., 183(3):311-341, 1983.

[3] H.W. Alt, S. Luckhaus, and A. Visintin. On nonstationary flow through porous media. Ann. Mat. Pura Appl. (4), 136:303-316, 1984.

[4] F. Bernis. Finite speed of propagation and continuity of the interface for thin viscous flows. Adv. Differential Equations, 1(3):337-368, 1996.

[5] M. Bertsch, R. Dal Passo, and C. J. van Duijn. Analysis of oil trapping in porous media flow. SIAM J. Math. Anal., 35(1):245-267 (electronic), 2003.

[6] Z. Chen. Degenerate two-phase incompressible flow. I. Existence, uniqueness and regularity of a weak solution. J. Differential Equations, 171(2):203-232, 2001. 
[7] Z. Chen. Degenerate two-phase incompressible flow. II. Regularity, stability and stabilization. J. Differential Equations, 186(2):345-376, 2002.

[8] R. Dal Passo, H. Garcke, and G. Grün. On a fourth-order degenerate parabolic equation: global entropy estimates, existence, and qualitative behavior of solutions. SIAM J. Math. Anal., 29(2):321-342 (electronic), 1998.

[9] C. J. van Duijn, A. Mikelić, and I. S. Pop. Effective equations for two-phase flow with trapping on the micro scale. SIAM J. Appl. Math., 62(5):1531-1568 (electronic), 2002.

[10] C.J. van Duijn, H. Eichel, R. Helmig, and I.S. Pop. Effective equations for twophase flow in porous media: the effect of trapping on the microscale. Transp. Porous Media, 69(3):411-428, 2007.

[11] D. Kröner and S. Luckhaus. Flow of oil and water in a porous medium. J. Differential Equations, 55(2):276-288, 1984.

[12] M. Lenzinger and B. Schweizer. Two-phase flow equations with outflow boundary conditions in the hydrophobic-hydrophilic case. Technical Report 2008-12, Fakultät für Mathematik, TU Dortmund, 2008.

[13] M. Ohlberger and B. Schweizer. Modelling of interfaces in unsaturated porous media. Discrete Contin. Dyn. Syst., (Dynamical Systems and Differential Equations. Proceedings of the 6th AIMS International Conference, suppl.):794-803, 2007.

[14] F. Otto. $L^{1}$-contraction and uniqueness for quasilinear elliptic-parabolic equations. J. Differential Equations, 131(1):20-38, 1996.

[15] B. Schweizer. Regularization of outflow problems in unsaturated porous media with dry regions. J. Differential Equations, 237(2):278-306, 2007.

[16] B. Schweizer. Homogenization of degenerate two-phase flow equations with oil trapping. SIAM J. Math. Anal., 39(6):1740-1763, 2008.

Fulvia Buzzi

via del Sole 19

CH-6943 Vezia, Switzerland
Michael Lenzinger and Ben Schweizer

Fakultät für Mathematik

TU Dortmund

Vogelpothsweg 87

D-44227 Dortmund, Germany

michael.lenzinger@tu-dortmund.de,

ben.schweizer@tu-dortmund.de 


\section{Preprints ab 2008}

2009-02

2009-01

2008-25

2008-24

2008-23

2008-22

2008-21

2008-20

2008-19

2008-18

2008-17

2008-16

$2008-15$
Fulvia Buzzi, Michael Lenzinger and Ben Schweizer

Interface conditions for degenerate two-phase flow equations

in one space dimension

Henryk Zähle

Approximation of SDEs by population-size-dependent

Galton-Watson processes

Winfried Hazod

MEHLER SEMIGROUPS, ORNSTEIN-UHLENBECK PROCESSES

AND BACKGROUND DRIVING LÉVY PROCESSES ON LOCALLY

COMPACT GROUPS AND ON HYPERGROUPS

Karl Friedrich Siburg, Pavel A. Stoimenov

Symmetry of functions and exchangeability of random variables

Ina Kirsten Voigt

Voronoi Cells of Discrete Point Sets

Michael Lenzinger and Ben Schweizer

Effective reaction rates of a thin catalyst layer

Michael Voit

Bessel convolutions on matrix cones: Algebraic properties and random walks

Margit Rösler and Michael Voit

Limit theorems for radial random walks on $p \times q$-matrices as

$p$ tends to infinity

Michael Voit

Central Limit Theorems for Radial Random Walks on

$p \times q$ Matrices for $p \rightarrow \infty$

\section{Michael Voit}

Limit theorems for radial random walks on homogeneous spaces with growing dimensions

Ansgar Steland and Henryk Zähle

Sampling inspection by variables: nonparametric setting

Guy Bouchitté and Ben Schweizer

Homogenization of Maxwell's equations with split rings

Wilfried Hazod

Multiple selfdecomposable laws on vector spaces and on groups:

The existence of background driving processes 
2008-14

2008-13

2008-12

2008-11

2008-10

2008-09

2008-08

2008-07

2008-06

2008-05

2008-04

2008-03

2008-01
Wilfried Hazod

Mixing of generating functionals and applications to (semi-)stability of probabilities on groups

Wilfried Hazod

Probability on Matrix-Cone Hypergroups: Limit Theorems and Structural Properties

Michael Lenzinger and Ben Schweizer

Two-phase flow equations with outflow boundary conditions in the hydrophobic-hydrophilic case

Karl Friedrich Siburg

Geometric proofs of the two-dimensional Borsuk-Ulam theorem

Peter Becker-Kern, Wilfried Hazod

Mehler hemigroups and embedding of discrete skew convolution

Karl Friedrich Siburg, Pavel A. Stoimenov

Gluing copulas

Karl Friedrich Siburg, Pavel A. Stoimenov

A measure of mutual complete dependence

Karl Friedrich Siburg, Pavel A. Stoimenov

A scalar product for copulas

Flavius Guiaş

Generalized Becker-Döring Equations Modeling the Time Evolution of a Process of Preferential Attachment with Fitness

Benjamin Fine, Alexei Myasnikov, Gerhard Rosenberger Generic Subgroups of Group Amalgams

\section{Ben Schweizer}

Homogenization of the Prager model in one-dimensional plasticity

Benjamin Fine, Miriam Hahn, Alexander Hulpke, Volkmar große Rebel, Gerhard Rosenberger, Martin Scheer All Finite Generalized Tetrahedron Groups

Benjamin Fine, Gerhard Rosenberger

An Epic Drama: The Development of the Prime Number Theorem

Henryk Zähle

Weak approximation of SDEs by discrete-time processes 